\title{
Ultrafast Coherent Electron Spin Flip in a Modulation-Doped CdTe Quantum Well
}

\author{
Carey Phelps, ${ }^{1}$ Timothy Sweeney, ${ }^{1}$ Ronald T. Cox,${ }^{2}$ and Hailin Wang ${ }^{1}$ \\ ${ }^{1}$ Department of Physics, University of Oregon, Eugene, Oregon 97403, USA \\ ${ }^{2}$ CEA/CNRS/UJF Joint Group "Nanophysics and Semiconductors," Institut Néel, Grenoble, France
}

(Received 2 January 2009; published 12 June 2009)

\begin{abstract}
We report the experimental realization of coherent electron spin flip in a modulation-doped CdTe quantum well. Coherent spin rotation is realized with an off-resonant laser pulse, which induces a polarization-dependent optical Stark shift in the trion resonance. Complete electron spin flip is made possible by a laser pulse designed to avoid excessive excitations of nearby exciton resonances and minimizes detrimental many-body effects. These results demonstrate an effective approach for ultrafast optical spin control in a complex spin system.
\end{abstract}

DOI: 10.1103/PhysRevLett.102.237402

The understanding and control of spin dynamics in individual and ensemble electron spins in semiconductors have attracted intense research efforts in recent years [1,2]. Individual spins can serve as qubits for quantum information processing. Ensemble spin systems can be exploited for a variety of applications such as spin-based electronic devices $[1,2]$, electromagnetically induced transparency [3], and light-matter quantum interfaces [4]. Coherent spin rotation with time scales faster than other dynamical processes is crucially important for many of these applications as well as for fundamental spectroscopic studies. Various resonant and off-resonant ultrafast optical spin rotation schemes have been analyzed theoretically [5-8].

While complete electron spin flips with ultrafast optical pulses have recently been demonstrated in single quantum dots (QDs) that feature atomiclike optical transitions $[9,10]$, only a limited degree of spin rotation has been achieved in ensemble spin systems including 2D electron gases (2DEGs), donor-bound electrons, and inhomogeneously broadened QDs [11-15]. Among the various ensemble spin systems, 2DEGs are especially interesting and challenging since, in addition to short decoherence times, coherent optical processes in a 2DEG are also strongly influenced by inherent many-body interactions among electrons, excitons, and trions.

In this Letter, we report the experimental demonstration of complete electron spin flips in a 2DEG using an offresonant ultrafast laser pulse (referred to as the control pulse). The coherent electron spin rotation takes place via a polarization-dependent optical Stark shift of the trion resonance [11]. Complete electron spin flip is demonstrated via a comparison of coherent spin dynamics before and after the arrival of the control pulse. The key to our experimental success is the use of a control pulse which avoids excessive excitations of nearby exciton resonances and thereby minimizes detrimental many-body effects. The experimental results on the electron spin flip are in good agreement with separate measurements of optical Stark shifts and with our theoretical analysis based on optical Bloch equations (OBEs).
PACS numbers: 78.67.De, 42.50.Ex, 71.35.Pq, 78.47.Fg

For a $2 \mathrm{DEG}$ in a quantum well $(\mathrm{QW})$ with growth axis along $z$, a $\sigma+($ or $\sigma-$ ) polarized optical field couples the electron state with $s_{z}=1 / 2$ (or $s_{z}=-1 / 2$ ) to trion state $\left|t_{+}\right\rangle$(or $\left|t_{-}\right\rangle$), which consists of a hole with $J_{z}=3 / 2$ (or $J_{z}=-3 / 2$ ) and two electrons with opposite spins, as shown in Fig. 1(a) [16]. In the presence of an in-plane external magnetic field along the $x$ axis, the electron spins precess in the $y-z$ plane with Larmor period $T_{B}$. In the limit that the optical pulse duration is short compared with $T_{B}$, spin precession occurring during the optical interaction can be ignored and the optical transition can still be approximated as that shown in Fig. 1(a) [7]. In this case, an offresonant $\sigma+$ polarized pulse induces an optical Stark shift

(a)

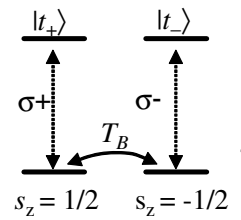

(b)

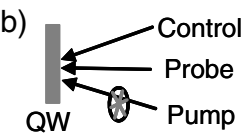

(c)
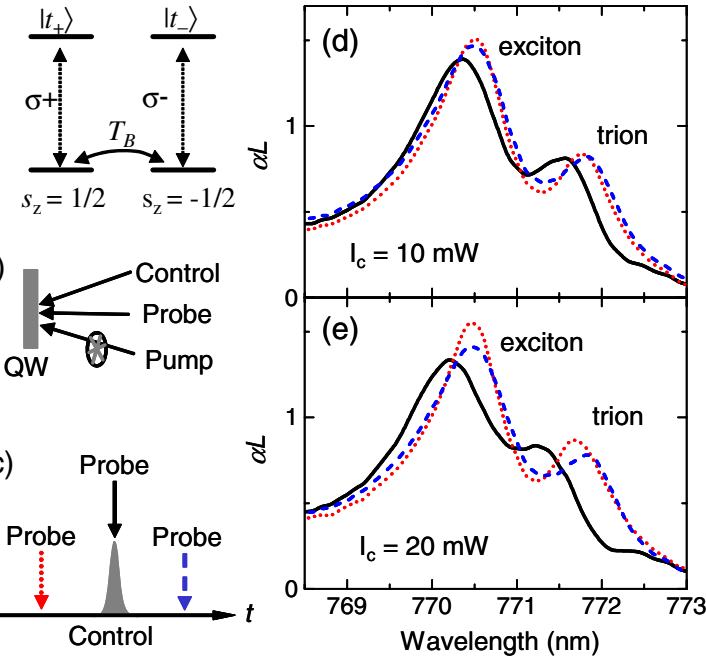

FIG. 1 (color online). (a) Schematic of the energy level structure of the trion transition, as discussed in the text. (b) Experimental setup for the spin rotation measurement. The pump intensity is modulated for lock-in detection. (c)-(e) Measurements of optical Stark shifts induced by the off-resonant control. The absorption spectra are obtained (in the absence of the pump) with the probe arriving at the peak of (solid curve), 20 ps before (dotted curve), and $20 \mathrm{ps}$ after (dashed curve) the control. The pulse timing is shown schematically in (c). 
for $\left|s_{z}=1 / 2\right\rangle$, resulting in a frequency separation, $\delta \omega(t)$, between $\left|s_{z}=1 / 2\right\rangle$ and $\left|s_{z}=-1 / 2\right\rangle$. The overall phase shift between these two spin states induced by the optical pulse is

$$
\phi=\int \delta \omega(t) d t
$$

This phase shift corresponds to a spin rotation of angle $\phi$ around the $z$ axis. For complete spin flip, $\delta \omega \sim \Omega^{2} / 2 \Delta$ and $\delta \omega \cdot \tau$ is of order $\pi$, where $\tau$ is the pulse duration, $\Delta$ is the detuning from the trion resonance, and $\Omega$ is the peak optical Rabi frequency.

At first glance, it appears that in order to avoid the excitation of the trion population and the decoherence occurring during the optical interaction, $\Delta \gg \gamma$ and $\tau \ll$ $1 / \gamma$, where $\gamma$ is the trion dipole decoherence rate, are needed for coherent spin flip. This, however, is not the case for a $2 \mathrm{DEG}$, which has a trion binding energy, $E_{b}$, only a few times $\hbar \gamma$. Strong optical coupling to nearby exciton resonances or electron-hole pairs and the resulting many-body effects have hindered earlier attempts to achieve the complete electron spin flip in 2DEGs [11,14].

To avoid the excitation of excitons and electron-hole pairs, one needs to minimize optical coupling to transitions other than the trion resonance. In a counterintuitive way, this dictates that $1 / \tau, \Delta$, and especially $\Omega$ should not greatly exceed $E_{b} / \hbar$. A suitable choice of control parameters, which can meet these requirements, is $\tau \sim 1 / \gamma$ and $\Delta \sim\left(E_{b} / \hbar\right)^{2} \tau / 2 \pi$, with $\Omega \sim E_{b} / \hbar$ for complete spin flip. In essence, $E_{b}$ is the key constraint for the design of the control pulse. Note that our own attempts of coherent electron spin flips in 2DEGs with off-resonant pulses featuring $\Omega \gg E_{b} / \hbar$ have not succeeded.

The experimental studies were carried out at $5 \mathrm{~K}$ in a high quality $n$-doped CdTe QW grown by molecular beam epitaxy on a $\mathrm{Cd}_{0.88} \mathrm{Zn}_{0.12}$ Te substrate that is transparent near the band edge. The sample consists of 10 periods of $10 \mathrm{~nm}$ CdTe wells and $45 \mathrm{~nm} \mathrm{Cd}{ }_{0.84} \mathrm{Zn}_{0.16}$ Te barriers [16]. The modulation doping density is estimated to be $3 \times$ $10^{10} / \mathrm{cm}^{2}$. At $10 \mathrm{~K}$, the trion resonance is characterized by a linewidth of $0.8 \mathrm{~nm}(1.6 \mathrm{meV})$ and a trion binding energy of $2.5 \mathrm{meV}(1.25 \mathrm{~nm})$.

For coherent spin rotation, we have chosen a control pulse which has a duration of 2 ps (bandwidth $0.5 \mathrm{~nm}$ ) and is only slightly detuned ( $2 \mathrm{~nm}$ or $4 \mathrm{meV}$ ) below the trion resonance, in contrast to the usual assumption of $\tau \ll 1 / \gamma$ and $\Delta \gg \gamma$. The control is added to a conventional pumpprobe setup, as shown in Fig. 1(b). In these experiments, the pump initializes via the trion transition an electron spin polarization along the $z$ axis [17-19]. The spin polarization precesses around the external magnetic field along the $x$ axis $(B=0.4 \mathrm{~T})$. A weak probe, arriving at a later time, detects the spin precession by measuring the differential transmission (DT) induced by the precessing spin polarization [19]. The control, applied at a fixed delay after the pump, induces an electron spin rotation around the $z$ axis.
All three laser pulses are derived from a femtosecond mode-locked Ti:sapphire laser (repetition rate $82 \mathrm{MHz}$ ) with grating-based spectral pulse shapers. After propagating through the sample, the spectrally broad probe is dispersed in a spectrometer. The change in the probe transmission induced by the pump is measured with lockin detection. The pump and probe have the opposite and the control and probe have the same circular polarization. The trion density excited by the pump is kept below a few times $10^{9} / \mathrm{cm}^{2}$ to avoid additional decoherence. The spot size for the pump, probe, and control is estimated to be $3 \times 10^{-5}$, $1 \times 10^{-6}, 2 \times 10^{-5} \mathrm{~cm}^{2}$, respectively. An average control power of $I_{c}=1 \mathrm{~mW}$ corresponds to an energy flux per pulse of $0.6 \mu \mathrm{J} / \mathrm{cm}^{2}$.

We have carried out detailed experimental studies to probe the changes in both trion and exciton resonances induced by the control. Ideally, for coherent spin rotation, the control should induce optical Stark shifts, but excite no real populations. Figures 1(d) and 1(e) show the probe absorption spectra obtained (in the absence of the pump) with the probe arriving at various delays relative to the peak of the control [see Fig. 1(c)]. A comparison of the absorption spectra when the probe arrives at 20 ps before and at the peak of the control show an optical Stark shift of 0.3 and $0.6 \mathrm{~nm}$ for the trion resonance with $I_{c}=10 \mathrm{~mW}$ and $I_{c}=20 \mathrm{~mW}$, respectively [20]. More importantly, a comparison of the absorption spectra when the probe arrives at 20 ps before and $20 \mathrm{ps}$ after the control shows that with $I_{c}=10 \mathrm{~mW}$ [see Fig. 1(d)] both the trion and exciton resonances recover nearly completely after the passage of the control and there is negligible bleaching in the absorption spectra. While excitations of exciton and trion populations are observed at $I_{c}=20 \mathrm{~mW}$ [see Fig. 1(e)], bleaching at both the exciton and trion resonances still remains relatively small. These studies further validate our choice of pulse parameters.

For transient DT studies, the excitation of $\left|t_{+}\right\rangle$by a $\sigma+$ -polarized pump leaves a net spin-polarized electron population with $s_{z}=-1 / 2$ in the 2D electron gas, generating a spin polarization initially along the $z$ axis [17-19]. Larmor precession of the spin polarization leads to spin beats with $T_{B}=116 \mathrm{ps}$, as shown by the top curve in Fig. 2. The phase of the spin beats indicates the direction of the spin polarization in the $y-z$ plane. The decay of the spin beats is determined by the spin dephasing time $T_{2}^{*} \sim 0.5 \mathrm{~ns}$ and has been investigated in extensive earlier studies [17-19].

Figure 2 shows the temporal evolution of the spin polarization when a control is applied at a delay of $1.75 T_{B}$ after the pump, at which time the spin polarization is along the $y$ axis. As illustrated by the Bloch vector in the inset of Fig. 2, the control rotates the spin polarization around the $z$ axis. The amplitude of the spin beats is then determined by the projection of the spin polarization in the $y-z$ plane. With increasing control power, the spin-beat amplitude varies according to $\cos (\phi)$, where $\phi$ is the spin rotation angle discussed earlier, while the phase of the spin beats 


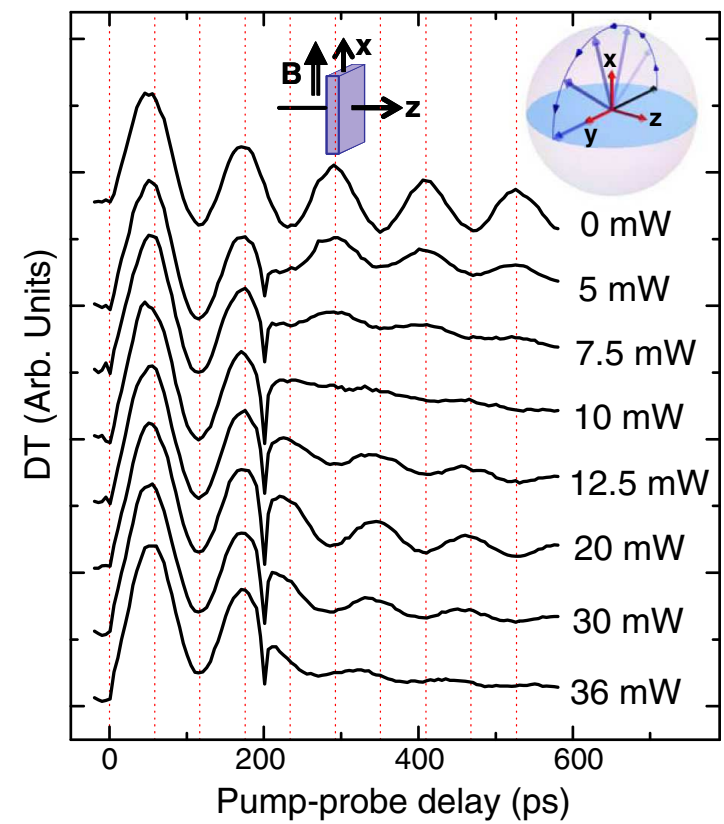

FIG. 2 (color online). Transient DT as a function of the pumpprobe delay and with the control applied at a delay of $1.75 T_{B}$ after the pump. The average control power is indicated in the figure. The Bloch sphere illustrates a spin polarization initially along the $y$ axis rotating around the $z$ axis.

remains either unchanged or changes by $\pi$ (with the exception of the DT response obtained with the highest $I_{c}$ in Fig. 2). At $I_{c}=10 \mathrm{~mW}$, the spin beats vanish, indicating that the spin polarization is rotated to the $x$ axis, for which there is no spin precession. In this case, the control pulse corresponds to a $\pi / 2$ rotation for the electron spin. At $I_{c}=$ $20 \mathrm{~mW}$, the control induces a spin flip. The spin rotation observed in Fig. 2 is in good agreement with the optical Stark shifts obtained from Fig. 1. The fidelity of the spin rotation and the non- $\pi$ phase shift observed at the highest $I_{c}$ in Fig. 2 will be discussed in detail later.

Figure 3(a) shows the temporal evolution of the spin polarization when a control with $I_{c}=20 \mathrm{~mW}$ is applied at various delays after the pump and also compares the spin evolution with that obtained in the absence of the control. The spin dynamics depend on the arrival time of the control. When the control is applied at a delay of $1.25 T_{B}$ or $1.75 T_{B}$ after the pump, at which time the spin polarization is along the $y$ axis, the control induces a phase change of $\pi$, as discussed earlier. In comparison, since the spin rotation is induced around the $z$ axis, there is no phase change when the control is applied at a delay of $1.5 T_{B}$ or $2 T_{B}$ after the pump, at which time the spin polarization is along the $z$ axis.

A unique signature of a complete electron spin flip is that the spin precession should be symmetric with respect to the arrival time of the spin-flip pulse, as illustrated in the inset of Fig. 3(b). Figure 3(b) shows transient DT responses obtained with $I_{c}=20 \mathrm{~mW}$ and at various fixed pumpcontrol delays. For clarity, the DT responses are plotted
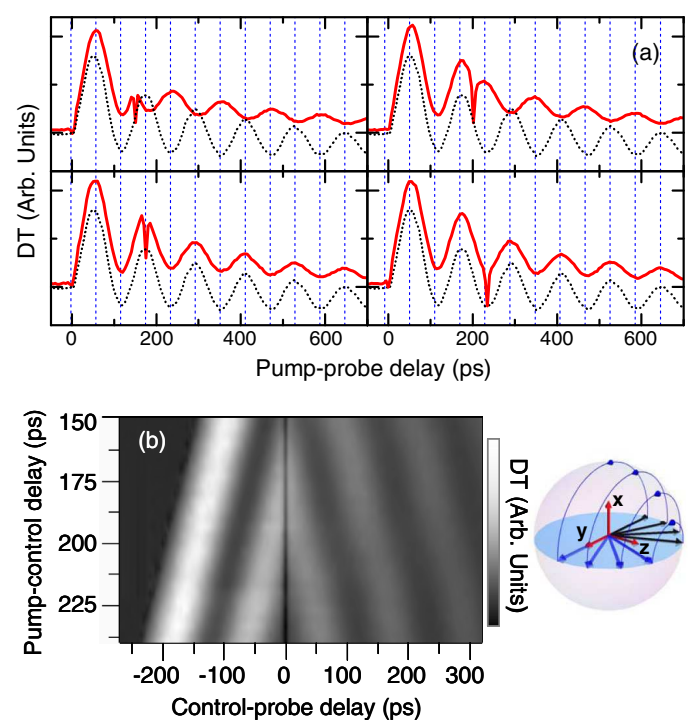

FIG. 3 (color online). Transient DT obtained with $I_{c}=$ $20 \mathrm{~mW}$. (a) As a function of the pump-probe delay with the control applied at a delay of $1.25 T_{B}, 1.5 T_{B}, 1.75 T_{B}$, and $2 T_{B}$ after the pump. The control timing can be seen from the spike in DT. Dashed curves show the DT obtained without the control. (b) As a function of the control-probe delay and at various fixed delays between the pump and control. The Bloch sphere illustrates spin flips around the $z$ axis.

as a function of the control-probe delay instead of the pump-probe delay. As shown in the figure, the phase of the spin precession is symmetric with respect to the controlprobe delay, regardless of the arrival time of the control. The symmetric spin dynamics not only represent a direct and remarkable manifestation of the complete spin flip induced by the control, but also demonstrate the feasibility of using the ultrafast optical pulse for electron spin echoes.

We have analyzed theoretically the electron spin rotation induced by an off-resonant control pulse by using the OBEs associated with the trion transitions [7]. For the calculation, the system is initialized such that at $t=0$ the spin population is all at state $\left|s_{z}=-1 / 2\right\rangle$. A 2 ps control pulse with a temporal Gaussian line shape is then applied at a detuning of $4 \mathrm{meV}$ below the trion resonance. Other parameters used include $\gamma=2 \mathrm{ps}^{-1}$, spin decoherence rate $\gamma_{s}=0.0025 \mathrm{ps}^{-1}$, and trion recombination rate $\Gamma=0.01 \mathrm{ps}^{-1}$, which is assumed to be small compared with the hole spin relaxation rate. For simplicity, we have ignored inhomogeneous broadening.

Figure 4 shows the calculated temporal evolution of the population difference between the $s_{z}= \pm 1 / 2$ spin states. The calculation describes well all the important features of the experimental results in Figs. 2 and 3. Figure 4(a) plots the dependence of the spin dynamics on the intensity of a control applied at $t_{\text {control }}=2.25 T_{B}$. Because of the dipole decoherence occurring during the optical coupling, the control excites a residual trion population that persists after the passage of the control. This residual trion population leads to the degradation in the fidelity of the spin rotation in 

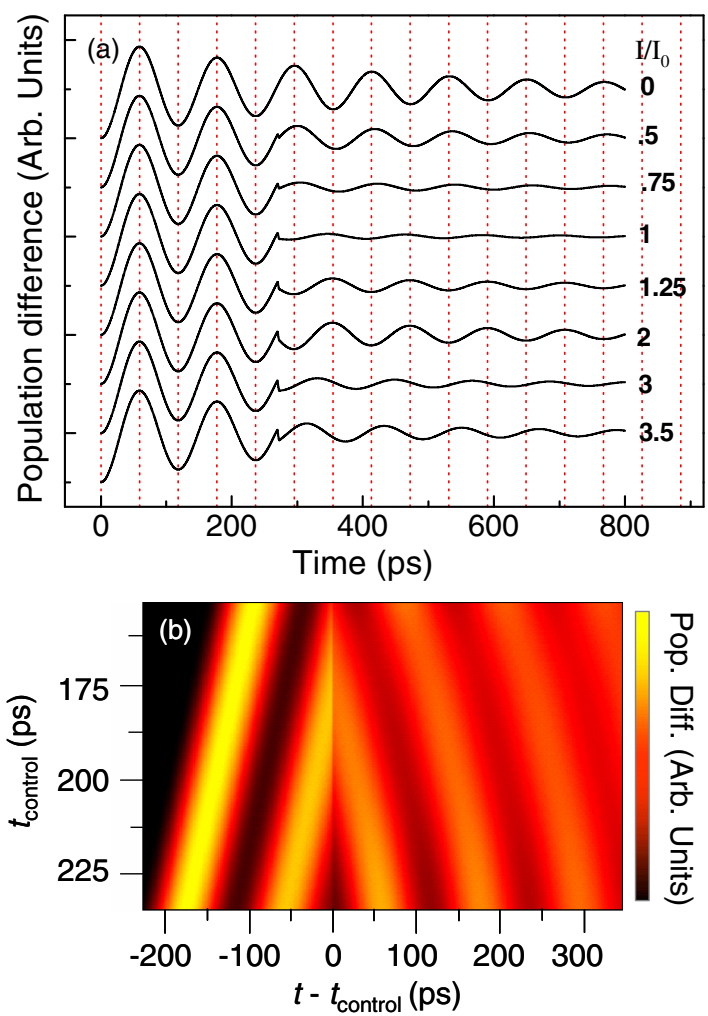

FIG. 4 (color online). Calculated temporal evolution of the population difference between $s_{z}= \pm 1 / 2$ states with parameters given in the text. (a) A control is applied at a delay of $2.25 T_{B}$. The average control intensity is indicated in the figure with $I_{0}$ the average intensity for the $\pi / 2$ pulse. (b) A control with $I=2 I_{0}$ is applied at various fixed delays.

Figs. 4(a) and 2 as well as to a small amount of bleaching in the trion resonance. The excitation of the residual trion population also initializes an additional spin polarization, which precesses in the $y$ - $z$ plane with a phase dependent on the timing of the control. A control with a relatively high intensity can thus induce a pronounced phase shift (other than $\pi$ ) in the spin precession, as shown in Figs. 4(a) and 2. It should also be noted that optical Stark shifts and thus the degree of spin rotation are no longer proportional to the control intensity when $\Omega$ exceeds or approaches $\Delta$.

Figure 4(b) plots the calculated dependence of the spin dynamics on the timing of the spin-flip control pulse, revealing a slight asymmetry with respect to the arrival time of the control. This asymmetry arises from the phase shift in the spin precession due to the spin polarization initialized by the control, as discussed above. The phase shift depends on the timing of the control, leading to the slightly curved pattern in Fig. 4(b). Note that the asymmetry is more pronounced in the theory than in the experiment since to underscore the effects of the residual trion population we have used in the calculation a dipole decoherence rate that significantly exceeds the actual decoherence rate (near $1 \mathrm{ps}^{-1}$ ).

In summary, by employing an off-resonant control pulse that induces an optical Stark shift for the trion resonance but avoids excessive excitation of nearby exciton resonances, we have successfully realized the complete spin flip of electrons in a 2DEG. The complete spin flip is demonstrated by spin precessions that are symmetric with respect to the arrival time of the control. The spinflip experiments are well described by a theoretical analysis based on the OBEs. Our results demonstrate an effective approach to realizing ultrafast optical spin control in a many-body spin system. The fidelity of the spin rotation can be further improved by exploiting quantum interference and coherent control techniques [21]. The experimental realization of ultrafast spin flip paves the way for further spectroscopic studies including spin echoes and the exploration of dynamical decoupling of electron spins from their surrounding environment with a series of ultrafast spin-flip pulses [22-24].

This work is supported by NSF DMR Grant No. 0804559 and by ARL.

Note added.-After submission of the Letter, we learned of ultrafast optical spin flip in ensemble quantum dots reported by Greilich et al. [25].

[1] Semiconductor Spintronics and Quantum Computation, edited by D. D. Awschalom, D. Loss, and N. Samarth (Springer-Verlag, Berlin, 2002).

[2] A. Zutic, J. Fabian, and S. Das Sarma, Rev. Mod. Phys. 76, 323 (2004).

[3] S. E. Harris, Phys. Today 50, No. 7, 36 (1997).

[4] H. J. Kimble, Nature (London) 453, 1023 (2008).

[5] P. Chen et al., Phys. Rev. B 69, 075320 (2004).

[6] A. Greilich et al., Phys. Rev. Lett. 96, 227401 (2006).

[7] S. E. Economou et al., Phys. Rev. B 74, 205415 (2006).

[8] S. M. Clark et al., Phys. Rev. Lett. 99, 040501 (2007).

[9] J. Berezovsky et al., Science 320, 349 (2008).

[10] D. Press et al., Nature (London) 456, 218 (2008).

[11] J. A. Gupta et al., Science 292, 2458 (2001).

[12] Y. Shen, A. Goebel, and H. Wang, Phys. Rev. B 75, 045341 (2007).

[13] Y. Wu et al., Phys. Rev. Lett. 99, 097402 (2007).

[14] S. G. Carter, Z. Chen, and S. T. Cundiff, Phys. Rev. B 76, 201308 (2007).

[15] K.-M. C. Fu et al., Nature Phys. 4, 780 (2008).

[16] K. Kheng et al., Phys. Rev. Lett. 71, 1752 (1993).

[17] E. A. Zhukov et al., Phys. Rev. B 76, 205310 (2007).

[18] Z. Chen et al., Phys. Rev. B 75, 115320 (2007).

[19] S. O'Leary and H. Wang, Phys. Rev. B 77, 165309 (2008).

[20] Optical Stark shifts are negligible when the control and probe have the opposite circular polarization.

[21] P. Chen, C. Piermarocchi, and L. J. Sham, Phys. Rev. Lett. 87, 067401 (2001).

[22] G. S. Uhrig, Phys. Rev. Lett. 98, 100504 (2007).

[23] W. Yao, R.-B. Liu, and L. J. Sham, Phys. Rev. Lett. 98, 077602 (2007).

[24] B. Lee, W. M. Witzel, and S. Das Sarma, Phys. Rev. Lett. 100, 160505 (2008).

[25] A. Greilich et al., Nature Phys. 5, 262 (2009). 